\title{
Frequency of discussing and documenting advance care planning in primary care: secondary analysis of a multicenter cross- sectional observational study
}

\author{
Jun Hamano ${ }^{1 *}$, Ai Oishi², Tatsuya Morita ${ }^{3}$ and Yoshiyuki Kizawa ${ }^{4}$
}

\begin{abstract}
Background: To improve the quality of advance care planning (ACP) in primary care, it is important to understand the frequency of and topics involved in the ACP discussion between patients and their family physicians (FPs).

Methods: A secondary analysis of a previous multicenter cross-sectional observational study was performed. The primary outcome of this analysis was the frequency of and topics involved in the ACP discussion between outpatients and FPs. In March 2017, 22 family physicians at 17 clinics scheduled a day to assess outpatients and enrolled patients older than 65 years who were recognized by FPs as having regular visits. We defined three ACP discussion topics: 1) future decline in activities of daily living (ADL), 2) future inability to eat, and 3) surrogate decision makers. FPs assessed whether they had ever discussed any ACP topics with each patient and their family members, and if they had documented the results of these discussions in medical records before patients were enrolled in the present study. We defined patients as being at risk of deteriorating and dying if they had at least 2 positive general indicators or at least 1 positive disease-specific indicator in the Japanese version of the Supportive and Palliative Care Indicators Tool.

Results: In total, 382 patients with a mean age of $77.4 \pm 7.9$ years were enrolled, and $63.1 \%$ were female. Seventy-nine patients (20.7\%) had discussed at least one ACP topic with their FPs. However, only 23 patients (6.0\%) had discussed an ACP topic with family members and their FPs, with the results being documented in their medical records. The topic of future ADL decline was discussed and documented more often than the other two topics. Patients at risk of deteriorating and dying discussed ACP topics significantly more often than those not at risk of deteriorating and dying (39.4\% vs. $16.8 \%, p<0.001)$.

Conclusion: FPs may discuss ACP with some of their patients, but may not often document the results of this discussion in medical records. FPs need to be encouraged to discuss ACP with patients and family members and describe the decisions reached in medical records.
\end{abstract}

Keywords: Advance care planning, Aged patients, End of life care, Family physician, Primary care outpatients, Supportive and palliative care indicators tool

* Correspondence: junhamano@md.tsukuba.ac.jp

'Division of Clinical Medicine, Faculty of Medicine, University of Tsukuba,

1-1-1 Tennoudai, Tsukuba, Ibaraki 305-8575, Japan

Full list of author information is available at the end of the article

(c) The Author(s). 2020 Open Access This article is licensed under a Creative Commons Attribution 4.0 International License, which permits use, sharing, adaptation, distribution and reproduction in any medium or format, as long as you give appropriate credit to the original author(s) and the source, provide a link to the Creative Commons licence, and indicate if changes were made. The images or other third party material in this article are included in the article's Creative Commons licence, unless indicated otherwise in a credit line to the material. If material is not included in the article's Creative Commons licence and your intended use is not permitted by statutory regulation or exceeds the permitted use, you will need to obtain permission directly from the copyright holder. To view a copy of this licence, visit http://creativecommons.org/licenses/by/4.0/. The Creative Commons Public Domain Dedication waiver (http://creativecommons.org/publicdomain/zero/1.0/) applies to the data made available in this article, unless otherwise stated in a credit line to the data. 


\section{Introduction}

Advance care planning (ACP) is an important process for sharing care preferences, discussing the goals of care, and making care plans through discussions between patients and health care providers $[1,2]$. A recent study revealed that appropriate ACP was beneficial for patients, their families, and the health system because it increases autonomy, dignity, peace, and intimacy at the time of death, lessens grieving and decreases the risk of mental health issues for family members, and reduces health care costs and the utilization of resources [3].

Previous studies indicated that early recognition of the risk of a particular patient deteriorating and dying is difficult, which suggests that the opportunity for ACP discussions needs to be provided at the right time from the perspective of illness trajectories $[4,5]$. Since illness trajectories vary widely, the development of ACP that considers an individual patient's trajectory is important [5].

In the opinion of patients, families, and health care professionals, the family physician (FP) is the key professional for the discussion of ACP [4, 6-9]. Previous studies investigated the attitude of FPs toward ACP and the facilitators of or barriers to discussing ACP with FPs [10]; limited information is currently available on frequency of and topics involved in the ACP discussion among patients, their family members, and FPs [11-17].

According to a previous mortality follow-back study, 34\% of 1072 patients with non-sudden death had discussed ACP with their FPs [16]. That study focused on terminally ill patients, and revealed that the most frequent topic of discussion was not adopting potential life-prolonging treatments. Another cross-sectional study revealed that ACP was discussed between $16.3 \%$ of outpatients and their FPs; however, the topics under discussion were not investigated [17]. In addition, a recent study based on hypothetical vignette scenarios showed that FPs were more likely to discuss ACP when patients had severe clinical manifestations [10]. A better understanding of the frequency of and topics involved in the ACP discussion as well as the characteristics of patients who discuss ACP with their FPs may contribute to improving ACP quality in the primary care setting.

Therefore, we herein performed a secondary analysis of our previous multicenter observational study to investigate the frequency of and topics involved in the ACP discussion among outpatients, their family members, and FPs involved in primary care. In addition, the frequency of the ACP discussion was compared between patients who were/were not at risk of deteriorating and dying, and the relationships between FP background factors and the discussion of ACP were examined.

\section{Methods}

The present study involved a secondary analysis of our previous multicenter cross-sectional observational study in Japan. Our previous study focused on the prevalence and characteristics of primary care outpatients at risk of deteriorating and dying [18]. Participating facilities and FPs were collected by purposive sampling. Each participating clinic provided ambulatory care for community residents and had at least one FP. In March 2017, 17 clinics (22 FPs) scheduled a day to assess their outpatients in advance. Patients who were older than 65 years and who were recognized by FPs as having regular visits were enrolled. The present study was approved by the Institutional Review Board of the University of Tsukuba (No. 1089).

\section{Advance care planning}

ACP was defined according to a previous Delphi study that indicated ACP as a process that supports adults of any age or health status to understand and share their personal values, life goals, and preferences regarding future medical care [2]. We also defined three ACP discussion topics, and developed ad hoc questionnaires based on a literature review and discussion among the authors $[1,2,11,12,14,16$, $17,19,20]$. The three ACP discussion topics were as follows: 1) future decline in the activities of daily living (ADL), 2) future inability to eat, and 3) surrogate decision makers. We defined the frequency of the ACP discussion as discussing any of these topics at least once among the patient and FP before enrollment in the study. Even when patients and FPs talked about multiple topics more than once, it was considered to be one ACP discussion. In addition, we defined an optimized ACP discussion as a discussion held among the patient, family members, and FP together with documentation of the results in medical records. This definition of an optimized ACP discussion was based on the international consensus definition of ACP, namely, ACP involves a patient discussing the abovementioned goals and preferences with family members and health care providers, followed by recording and reviewing the patient's preferences if appropriate [1].

\section{Patients at risk of deteriorating and dying}

We identified patients who were at risk of deteriorating and dying using the Japanese version of the Supportive and Palliative Care Indicators Tool (SPICT-JP), as previously reported [18]. The original Supportive and Palliative Care Indicators Tool (SPICT ${ }^{\mathrm{TM}}$ ) was developed to identify patients whose health condition is deteriorating. Details of the development of SPICT ${ }^{\mathrm{TM}}$ and SPICT-JP have been described elsewhere [18, 21-25].

\section{Data collection}

FPs were asked whether they had ever discussed any of the ACP topics (a patient's future ADL decline, future inability to eat, and surrogate decision makers) with each patient and their family members before the patient was enrolled in the present study based on the FP's memory, 
and to confirm whether the discussion had been documented in medical records. FPs also assessed patients according to the Palliative Performance Scale (PPS) [26], and 6 general clinical indicators and 25 disease-specific indicators in the SPICT-JP (Additional file 1). Furthermore, FPs recorded the demographic and clinical characteristics of patients. We also investigated the background factors of FPs, including sex, duration of clinical practice after obtaining a medical license, experience with a palliative care unit, and participation in the nationwide palliative care education program.

\section{Statistical analysis}

Descriptive statistics were calculated for each ACP discussion topic. According to previous studies, we defined patients as being at risk of deteriorating and dying if they had at least 2 positive general indicators or at least 1 positive disease-specific indicator in the SPICT-JP [25]. The relationship between the discussion of ACP and a patient's risk of deteriorating and dying was examined. We also investigated the relationship between the discussion of ACP and patients with PPS $\leq 70$. The characteristics of participants were reported as proportions for categorical variables and were analyzed by Pearson's $\chi^{2}$ test or Fisher's exact test, while continuous variables were analyzed by the Student's $t$-test. To examine the relationship between FP background factors and the discussion of ACP topics, we performed univariate and multivariate logistic regression analyses to calculate the odds ratio (OR) and 95\% confidence interval ( $95 \% \mathrm{CI})$. FPs were categorized into 2 groups based on the duration of clinical practice ( $>15$ years or $\leq 15$ years). Background factors of FPs that showed significance in univariate analyses were employed as independent variables in multivariate analyses. SPSS-J software (version 24.0; IBM, Tokyo, Japan) was used to conduct all analyses, and $p<0.05$ was considered to be significant.

\section{Results}

In total, 382 patients from 17 clinics (22 FPs) were included. Their mean age was $77.4 \pm 7.9$ years, and $63.1 \%$ were female. Most patients had PPS $\geq 80$ (79.1\%), and did not use care services (81.4\%). The main underlying diseases were hypertension (31.9\%), dementia/frailty (15.2\%), and cardiovascular disease, excluding hypertension (9.2\%) (Table 1).

Among the 22 participating physicians, 12 had trained at a palliative care unit (54.5\%) and 17 had participated in the nationwide palliative care education program (77.3\%) [27, 28] (Additional file 2).

\section{Frequency of the ACP discussion and topics}

While $20.7 \%$ of patients had discussed at least one of the ACP topics with their FPs, only $6.0 \%$ had participated in an optimized ACP discussion (Table 2).
Table 1 Demographic and Clinical Characteristics of Patients $(n=382)$

\begin{tabular}{|c|c|c|}
\hline & $\mathrm{n}$ & $\%$ \\
\hline Age (mean \pm standard deviation) & $77.4 \pm 7.9$ & \\
\hline \multicolumn{3}{|l|}{ Sex } \\
\hline Male & 141 & 36.9 \\
\hline Female & 241 & 63.1 \\
\hline \multicolumn{3}{|l|}{ Living situation } \\
\hline Living with family & 298 & 78.0 \\
\hline Living alone & 59 & 15.4 \\
\hline Care facility & 8 & 2.1 \\
\hline \multicolumn{3}{|l|}{ Main underlying disease } \\
\hline Hypertension & 122 & 31.9 \\
\hline Dementia/frailty & 58 & 15.2 \\
\hline Cardiovascular disease (excluding hypertension) & 38 & 9.9 \\
\hline Diabetes & 30 & 7.9 \\
\hline Hyperlipidemia & 19 & 5.0 \\
\hline Neurological disease & 18 & 4.7 \\
\hline Cancer & 14 & 3.7 \\
\hline Respiratory disease & 13 & 3.4 \\
\hline Musculoskeletal disease & 8 & 2.1 \\
\hline Mental disorder & 6 & 1.6 \\
\hline Gastroesophageal reflux disease & 6 & 1.6 \\
\hline Kidney disease & 5 & 1.3 \\
\hline Liver disease & 3 & 0.8 \\
\hline Others & 42 & 11.0 \\
\hline \multicolumn{3}{|l|}{ Palliative performance scale } \\
\hline 100 & 202 & 52.9 \\
\hline 90 & 51 & 13.4 \\
\hline 80 & 49 & 12.8 \\
\hline 70 & 20 & 5.2 \\
\hline 60 & 33 & 8.6 \\
\hline 50 & 22 & 5.8 \\
\hline 40 & 5 & 1.3 \\
\hline \multicolumn{3}{|l|}{ Current use of care services } \\
\hline No care service & 311 & 81.4 \\
\hline One or more care services & 71 & 18.6 \\
\hline \multicolumn{3}{|l|}{ Types of care services used (Multiple answers) ${ }^{a}$} \\
\hline Home visit nursing & 11 & 2.9 \\
\hline Nursing care services & 16 & 4.2 \\
\hline Home visit pharmacist & 1 & 0.3 \\
\hline Day care service & 54 & 14.1 \\
\hline Specialized palliative care service & 2 & 0.5 \\
\hline
\end{tabular}

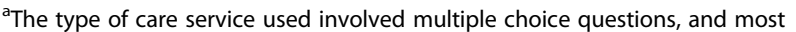
patients did not use the care services 


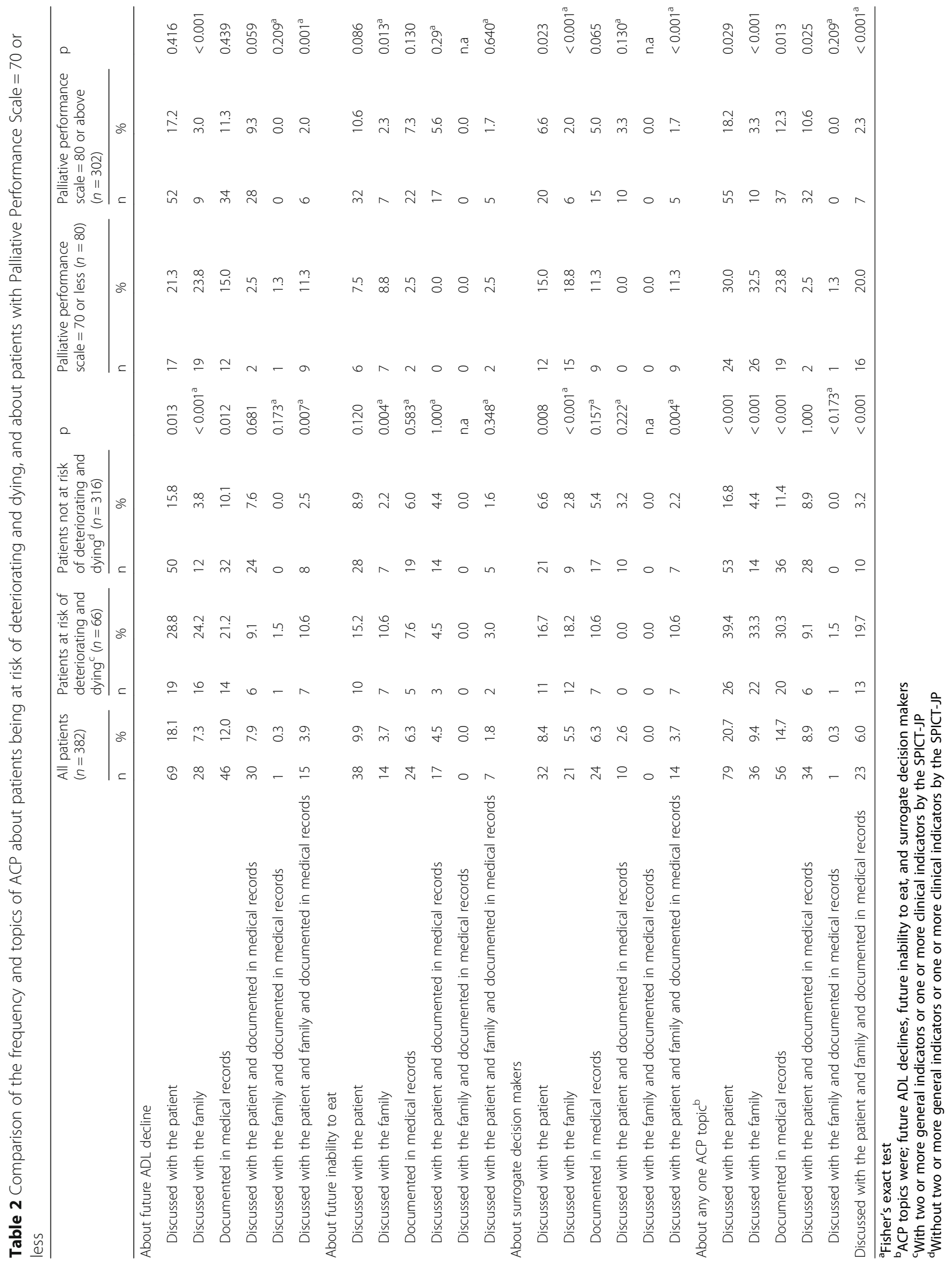


Table 3 Prevalence of patients at risk of deteriorating and dying

General clinical risk of deteriorating health ( $n=382$ )
Two or more unplanned hospital admissions in the
past 6 months
Performance status is poor or deteriorating, with
limited reversibility
Dependent on others for care due to physical and/or
mental health issues
Significant weight loss over the past 3-6 months
and/or a low body mass index
Persistent symptoms despite optimal treatment of
the underlying condition(s)
The patient or family asked for palliative care, treatment
withdrawal/limitation, or a focus on quality of life
Disease-specific risk for the deterioration of the conditions
Cancer ( $n=14$ )
Functional ability deteriorating due to progressive cancer
Too frail for cancer treatment or treatment for symptom
control
Dementia/frailty ( $n=56$ )
Unable to dress, walk, or eat without help
Eating and drinking less; difficulty swallowing
Urinary and fecal incontinence
No longer able to communicate using verbal language;
little social interaction
Fractured femur; multiple falls
Recurrent febrile episodes or infections; aspiration
pneumonia

Neurological disease $(n=18)$

Progressive deterioration of physical and/or cognitive function despite optimal therapy

Speech problems with increasing difficulty communicating and/or progressive difficulty swallowing

Recurrent aspiration pneumonia; breathless or respiratory failure

Cardiovascular disease $(n=38)$

NYHA Class III/IV heart failure or extensive, untreatable coronary artery disease with breathlessness or chest pain at rest or on minimal exertion

Severe, inoperable peripheral vascular disease

Respiratory disease $(n=13)$

Severe chronic lung disease with breathlessness at rest or on minimal exertion between exacerbations

Needs long-term oxygen therapy

Has needed ventilation for respiratory failure or ventilation is contraindicated

Kidney disease $(n=5)$

Stage 4 or 5 chronic kidney disease (eGFR $<30 \mathrm{ml} / \mathrm{min}$ ) with deteriorating health

Kidney failure complicating other life-limiting conditions or treatments
Table 3 Prevalence of patients at risk of deteriorating and dying (Continued)

n $\%$

10.3

246.3

266.8

$18 \quad 4.7$

$16 \quad 4.2$

$97 \quad 25.4$

\begin{tabular}{lll} 
& $\mathrm{n}$ & $\%$ \\
\hline Stopping dialysis & 0 & 0.0
\end{tabular}

Liver disease $(n=3)$

Advanced cirrhosis with one or more complications

in the past year: diuretic-resistant ascites

Advanced cirrhosis with one or more complications

$0 \quad 0.0$

in the past year: hepatic encephalopathy

Advanced cirrhosis with one or more complications

in the past year: hepatorenal syndrome

Advanced cirrhosis with one or more complications

in the past year: bacterial peritonitis

Advanced cirrhosis with one or more complications in the past year: recurrent variceal bleeds

Liver transplantation is contraindicated

$2 \quad 66.7$

Patients with liver disease had never discussed any of
$3 \quad 21.4$

214.3

$12 \quad 21.4$

$\begin{array}{ll}9 & 16.1\end{array}$

$13 \quad 23.2$

$23 \quad 41.1$

$10 \quad 17.9$

23.6

$7 \quad 38.9$

$2 \quad 11.1$

15.6

$4 \quad 10.5$

0.0

$2 \quad 15.4$

7.7

0.0

$3 \quad 60.0$

360.0 the ACP topics. On the other hand, one third of patients with cancer and neurological diseases, and one fifth of those with dementia/frailty had discussed at least one topic (Additional file 3). Each of the three ACP topics was discussed more frequently with patients (8.4-18.1\%) than with their family members (3.7-7.3\%) (Table 2). The topic of future ADL decline was discussed and documented more often than the other two topics. In 56 out of 79 patients $(70.9 \%)$ who had discussed an ACP topic, the discussion was documented in their medical records.

\section{Distribution of indicators for deteriorating and dying}

The distribution of general and specific indicators for deteriorating health based on the SPICT-JP is shown in Table 3. The most frequent general indicator was "The patient or family asked for palliative care, treatment withdrawal/limitation, or a focus on quality of life" (25.4\%). Based on our definition, 66 patients (17.3\%) were identified as being at risk of deteriorating and dying.

\section{Frequency of the ACP discussion and topics among} patients at risk of deteriorating and dying, and patients with PPS $\leq 70$

Table 2 shows the frequency of the ACP discussion and discussion topics among patients at risk of deteriorating and dying and patients with $\mathrm{PPS} \leq 70$. These patients discussed any one of the ACP topics significantly more often than those not at risk of deteriorating and dying (39.4\% vs. $16.8 \%, p<0.001)$, and also had a significantly higher frequency of an optimized ACP discussion (19.7\% vs. $3.2 \%, \mathrm{p}<0.001)$.

The topics of future ADL decline and surrogate decision makers were discussed significantly more often with patients at risk of deteriorating and dying than with those who were not at risk (28.8\% vs. $15.8 \%, p=0.013$; $16.7 \%$ vs. $6.6 \%, p=0.008)$; however, no significant 
differences were observed in future inability to eat (15.2\% vs. $8.9 \%, p=0.12$ ). An optimized ACP discussion on future ADL decline and surrogate decision makers was significantly more frequent among patients at risk of deteriorating and dying than among those not at risk (10.6\% vs. $2.5 \%, p=0.007 ; 10.6 \%$ vs. $2.2 \%, p=0.004$ ).

Patients with PPS $\leq 70(n=80)$ discussed any ACP topic significantly more often than those with PPS $\geq 80$ (30.0\% vs. $18.2 \%, p=0.029)$, and had a significantly higher frequency of the optimized ACP discussion (20.0\% vs. $2.3 \%, p<0.001)$.

\section{FP backgrounds and discussion of ACP}

According to the univariate analysis, male sex $(\mathrm{OR}=8.0$, $95 \%$ CI 1.9-33.5, $p=0.001)$, clinical practice for $\geq 15$ years $(\mathrm{OR}=2.1,95 \% \mathrm{CI} 1.3-3.4, p=0.005)$, training in a palliative care unit $(\mathrm{OR}=2.1,95 \% \mathrm{CI} 1.3-3.5, \mathrm{p}=0.005)$, and participation in the nationwide palliative care education program $(\mathrm{OR}=2.1,95 \% \mathrm{CI} 1.0-4.3, p=0.044)$ correlated with the discussion of ACP topics. The multivariate analysis confirmed that male sex $(\mathrm{OR}=6.6,95 \% \mathrm{CI} 1.5-29.3$, $p=0.012)$, clinical practice for $\geq 15$ years $(\mathrm{OR}=1.9$, $95 \%$ CI $1.1-3.3, p=0.021)$, and training in a palliative care unit $(\mathrm{OR}=2.6,95 \% \mathrm{CI} 1.4-4.6, p=0.002)$ correlated with the discussion of ACP topics (Additional file 4).

\section{Discussion}

To the best of our knowledge, this is the first large-scale cross-sectional survey on the frequency of discussing ACP and the topics involved among primary care outpatients, their family members, and FPs. The first important result was that $20.7 \%$ of primary care outpatients aged $>65$ years had discussed at least one ACP topic with their FPs. However, only $6 \%$ of patients had discussed at least one topic with their family members and FPs with documentation of the discussion in their medical records.

Since the frequency of discussing ACP depends on patient background factors and the definition of the discussion, a systematic review revealed that its frequency among frail elderly patients ranged widely between 2 and 29\% [13]. When limited to studies with a similar patient background and definition of discussing ACP, the present results were consistent with a multicenter crosssectional study on primary care outpatients in Japan, in which the frequency of discussing ACP was 16.2\% [17]. Therefore, approximately one fifth of primary care outpatients may participate in ACP discussions on future health care or surrogate decision makers with their FPs. It is difficult to judge whether this represents an appropriate discussion of ACP because the quality of end-oflife care was not assessed in the present study.

ACP discussions among patients, family members, and FPs with documentation in medical records, which we defined as an optimized ACP discussion, have not yet been examined. Considering the purpose of discussing ACP, it appears to be important to perform the optimized discussion in order to improve the quality of end-of-life care. We found that only $6.0 \%$ of patients had the optimized ACP discussion, suggesting effective ACP discussion processes have not yet been implemented in primary care.

The second important result of the present study was that almost one fifth of patients had discussed future ADL decline with their FPs. Although few studies have investigated the frequency of ACP discussion topics in the primary care setting, this result is similar to a previous finding showing that only $17 \%$ of community dwelling persons older than 80 years had discussed their wishes for end-of-life care with a physician or health care provider [20].

On the other hand, $42 \%$ of patients with mild dementia discussed illness-related topics, while 34\% discussed preferences for medical treatment with their FPs during the last 3 months of life [11]. This difference from the present results may be attributed to the frequency of discussing ACP increasing with the risk of deteriorating and dying because a previous study using hypothetical vignette scenarios revealed that FPs identified patients with severe clinical manifestations as needing the ACP discussion [10].

The third important result was that the discussion of ACP topics was significantly more frequent among patients at risk of deteriorating and dying than among those who were not at risk. This result is consistent with the study based on hypothetical vignette scenarios, which revealed that FPs were more likely to discuss ACP with patients showing severe clinical features [10]. Since the SPICT-JP was not assessed in the present study, our results may reflect the opinions of FPs on the risk of deteriorating and dying for primary care outpatients.

It is important to note that patients with PPS $\leq 70$ had a significantly higher proportion of ACP discussions with patient $\underline{(p}=0.029)$. However, the proportion of discussions with patients documented in medical records about any one of the ACP topics was significantly lower in patients with PPS $\leq 70 \underline{(p=0.025)}$, whereas no significant difference was observed among patients at risk of deteriorating and dying or not $(\underline{p}=1.000)$. This result suggests that FPs conduct an $\mathrm{ACP}$ discussion based on factors other than a poor performance status, such as a patient or family member asking for palliative care, treatment withdrawal/limitation, or a focus on quality of life.

The fourth important result was that male FPs with long clinical experience and training at palliative care units were more proactive about discussing ACP; however, the present results were not adjusted for variables such as the perception of ACP and end-of-life care. This result is consistent with the findings of a systematic review, which indicated that accumulated skills facilitate the engagement of FPs in ACP discussions [29]. While 
the present study suggested that male FPs were more frequently involved in ACP discussions, Fulmer et al. reported that female physicians were more likely to have these discussions [30]. This difference may have arisen because Fulmer's study included physicians from several specialties working in the hospital setting as well as FPs. Thus, further studies are needed to investigate the background factors of FPs that influence discussions of ACP in the primary care setting.

The present study had several limitations. We targeted a very small proportion of certified FPs in Japan by purposive sampling; therefore, the results obtained may not be representative and their interpretation requires caution. Furthermore, the present results may have been influenced by the Japanese health care system and cultural background; therefore, difficulties are associated with generalizing these results to other countries. In addition, observer recall bias may have influenced the data obtained because difficulties are associated with ascertaining whether undocumented ACP discussions occurred. A gap may exist in the perception of the ACP discussion between FPs and their patients. Another limitation is that there is currently no consensus that the three ACP discussion topics defined in the present study are standard evaluation items of the ACP process and outcomes. Therefore, caution is required when assessing the ACP process and outcomes performed by FPs based on the results of this study.

\section{Conclusion}

FPs may only discuss ACP with a few of their patients; however, this discussion may be more frequent with patients who are at risk of deteriorating and dying. The topic of future ADL decline was discussed and documented more often than other topics. However, FPs may not document the results of most ACP discussions in medical records. Further investigations are needed to establish whether the discussion of ACP between patients and FPs improves the quality of end-of-life care.

\section{Supplementary information}

Supplementary information accompanies this paper at https://doi.org/10. 1186/s12904-020-00543-y.

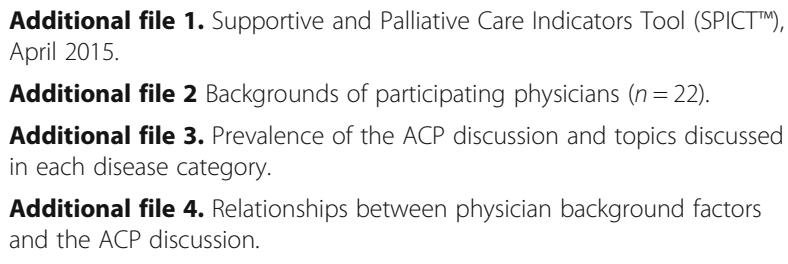

Additional file 4. Relationships between physician background factors and the ACP discussion.

\section{Abbreviations}

ACP: Advance care planning; FPs: Family physicians; SPICT-JP: The Japanese version of the Supportive and Palliative Care Indicators Tool; SPICT: The Supportive and Palliative Care Indicators Tool; PPS: Palliative Performance Scale; OR: Odds ratio; 95\% Cl: 95\% confidence interval

\section{Acknowledgements}

The participating study sites and investigators were: Hiroshi Takagi, M.D., Ph.D. (Kawasaki settlement Clinic), Takahiro Otsuka, M. D (Akito Otsuka Clinic), Makoto Kaneko M.D., PhD (Department of Family and Community Medicine, Hamamatsu University School of Medicine), Tetsuya Kanno, M. D, Hirotoshi Sasanuma, M. D (Aonohara Clinic), Gorou Hoshi, M. D (Hoshi Yokotsuka Clinic), Toshiharu Kitamura, M. D (Hobara Cyuou Clinic), Satochi Kanke, M.D., Ph.D. (Department of Family Medicine, Fukushima Medical University), Hiroshi Taira, M. D (Torimachi Clinic), Daisuke Kurihara, M. D (Kamakura Family Clinic), Tesshu Kusaba, M. D, Koutarou Satou, M. D (Motowanishi Clinic), Akihiro Imae, M. D (Suttu Clinic), Satoko Munakata, M. D (Sarashina-Mura Kokuho Clinic), Hiroki Ohashi, M. D, Mitsuru Takagi, M. D, Ken Horikoshi, M. D (Tama Family Clinic), Sachiko Ozone, M.D., Ph.D. (University of Tsukuba), Yousuke Kimura, M. D (Yamato Clinic), Yukihiro Sekiguchi, M. D, and Ayumi Yamada, M. D (Saiwai Clinic).

\section{Authors' contributions}

All authors made substantial contributions to the conception and design of the work. JH and AO facilitated the acquisition of data, TM and YK led the interpretation of data and drafting the work, all authors revised it critically, and all authors read and approved the final version of the manuscript.

\section{Funding}

This project received funding from the Japan Hospice/Palliative Care Foundation. The funder had no role in the design and conduct of the study: the collection, management, analysis, and interpretation of data; the preparation, review, and approval of the manuscript; or the decision to submit the manuscript for publication.

\section{Availability of data and materials}

The datasets used and/or analyzed during the present study are available from the corresponding author on reasonable request.

\section{Ethics approval and consent to participate}

The Institutional Review Board of the University of Tsukuba approved this study (No. 1089). Data collection was conducted by clinic staff who routinely access patient notes, and all data were anonymized prior to sharing with the research team.

\section{Consent for publication \\ Not applicable.}

\section{Competing interests}

The authors have declared that they have no competing interests.

\section{Author details}

'Division of Clinical Medicine, Faculty of Medicine, University of Tsukuba, 1-1-1 Tennoudai, Tsukuba, Ibaraki 305-8575, Japan. ${ }^{2}$ Primary Palliative Care Research Group, Usher Institute of Population Health Sciences and Informatics, University of Edinburgh, Medical School (Doorway 1), Teviot Place, Edinburgh, UK. ${ }^{3}$ Department of Palliative and Supportive Care, Palliative Care Team, and Seirei Hospice, Seirei Mikatahara General Hospital, 3453 Mikatahara-cho, Kita-ku, Hamamatsu, Shizuoka, Japan. ${ }^{4}$ Department of Palliative Medicine, Kobe University Graduate School of Medicine, Kobe, Hyogo, Japan.

Received: 20 July 2019 Accepted: 10 March 2020

Published online: 17 March 2020

\section{References}

1. Rietjens JAC, Sudore RL, Connolly M, et al. Definition and recommendations for advance care planning: an international consensus supported by the European Association for Palliative Care. Lancet Oncol. 2017;18(9):e543-51.

2. Sudore RL, Lum HD, You JJ, et al. Defining Advance Care Planning for Adults: A Consensus Definition From a Multidisciplinary Delphi Panel. J Pain Symptom Manage. 2017:53(5):821-32 e1.

3. Lum HD, Sudore RL, Bekelman DB. Advance care planning in the elderly. Med Clin North Am. 2015;99(2):391-403.

4. Mitchell S, Loew J, Millington-Sanders C, Dale J. Providing end-of-life care in general practice: findings of a national GP questionnaire survey. $\mathrm{Br} J \mathrm{Gen}$ Pract. 2016;66(650):e647-53. 
5. Evans N, Pasman HRW, Donker GA, et al. End-of-life care in general practice: a cross-sectional, retrospective survey of 'cancer', 'organ failure' and 'oldage/dementia' patients. Palliat Med. 2014;28(7):965-75.

6. Malcomson $\mathrm{H}$, Bisbee S. Perspectives of healthy elders on advance care planning. J Am Acad Nurse Pract. 2009;21(1):18-23.

7. Michiels E, Deschepper R, Van Der Kelen G, et al. The role of general practitioners in continuity of care at the end of life: a qualitative study of terminally ill patients and their next of kin. Palliat Med. 2007;21(5):409-15.

8. Oosterink JJ, Oosterveld-Vlug MG, Glaudemans JJ, Pasman HRW, Willems DL, Onwuteaka-Philipsen BD. Interprofessional communication between oncologic specialists and general practitioners on end-of-life issues needs improvement. Fam Pract. 2016;33(6):727-32.

9. De Vleminck A, Batteauw D, Demeyere T, Pype P. Do non-terminally ill adults want to discuss the end of life with their family physician? An explorative mixed-method study on patients' preferences and family physicians' views in Belgium. Fam Pract. 2018;35(4):495-502.

10. Sinclair C, Gates K, Evans S, Auret KA. Factors Influencing Australian General Practitioners' Clinical Decisions Regarding Advance Care Planning: A Factorial Survey. J Pain Symptom Manage. 2016;51(4):718-27 e2..

11. Miranda R, Penders YWH, Smets T, et al. Quality of primary palliative care for older people with mild and severe dementia: an international mortality follow-back study using quality indicators. Age Ageing. 2018;47(6):824-33.

12. Penders $Y W$, Van den Block $L$, Donker GA, Deliens L, Onwuteaka-Philipsen B, EURO IMPACT. Comparison of end-of-life care for older people living at home and in residential homes: a mortality follow-back study among GPs in the Netherlands. Br J Gen Pract. 2015;65(640):e724-30.

13. Sharp T, Moran E, Kuhn I, Barclay S. Do the Elderly Have a Voice? Advance Care Planning Discussions with Frail and Older Individuals: A Systematic Literature Review and Narrative Synthesis. Br J Gen Pract. 2013;63:e657-68.

14. Evans N, Pasman HR, Vega Alonso T, et al. End-of-Life Decisions: A CrossNational Study of Treatment Preference Discussions and Surrogate DecisionMaker Appointments. Smith TA, ed. PLoS One. 2013;8(3):e57965.

15. Howard M, Bernard C, Klein D, et al. Older patient engagement in advance care planning in Canadian primary care practices: results of a multisite survey. Can Fam Physician. 2018;64(5):371-7.

16. Meeussen $\mathrm{K}$, Van den Block $L$, Echteld $M$, et al. Advance care planning in Belgium and the Netherlands: a nationwide retrospective study via sentinel networks of general practitioners. J Pain Symptom Manag. 2011;42(4):565-77.

17. Aoki T, Miyashita J, Yamamoto Y, et al. Patient experience of primary care and advance care planning: a multicentre cross-sectional study in Japan. Fam Pract. 2017;34(2):206-12

18. Hamano J, Oishi A, Kizawa Y. Prevalence and Characteristics of Patients Being at Risk of Deteriorating and Dying in Primary Care. J Pain Symptom Manage. 2019;57(2):266-72 e1.

19. Evans N, Costantini M, Pasman HR, et al. End-of-Life Communication: A Retrospective Survey of Representative General Practitioner Networks in Four Countries. J Pain Symptom Manage. 2014;47(3):604-619.e3.

20. McCarthy EP, Pencina MJ, Kelly-Hayes M, et al. Advance care planning and health care preferences of community-dwelling elders: the Framingham heart study. J Gerontol A Biol Sci Med Sci. 2008;63(9):951-9.

21. Highet G, Crawford D, Murray SA, Boyd K. Development and evaluation of the supportive and palliative care indicators tool (SPICT): a mixed-methods study. BMJ Support Palliat Care. 2014;4(3):285-90.

22. Lynn J. Perspectives on care at the close of life. Serving patients who may die soon and their families: the role of hospice and other services. JAMA. 2001;285(7):925-32.

23. Boyd K, Murray SA. Recognising and managing key transitions in end of life care. BMJ. 2010;341:c4863.

24. Beaton D, Bombardier C, Guillemin F, Ferraz MB. Recommendations for the cross cultural adaptation of health status measures; 2002.

25. Hamano J, Oishi A, Kizawa Y. Identified palliative care approach needs with SPICT in family practice: a preliminary observational study. J Palliat Med. 2018;21(7):992-8.

26. Chan $\mathrm{E}-\mathrm{Y}, \mathrm{Wu} \mathrm{H}-\mathrm{Y}$, Chan $\mathrm{Y}-\mathrm{H}$. Revisiting the palliative performance scale: change in scores during disease trajectory predicts survival. Palliat Med. 2013;27(4):367-74

27. Yamamoto R, Kizawa Y, Nakazawa Y, Ohde S, Tetsumi S, Miyashita M. Outcome evaluation of the palliative care emphasis program on symptom management and assessment for continuous medical education: Nationwide physician education project for primary palliative Care in Japan. J Palliat Med. 2015;18(1):45-9.
28. Nakazawa Y, Yamamoto R, Kato M, Miyashita M, Kizawa Y, Morita T. Improved knowledge of and difficulties in palliative care among physicians during 2008 and 2015 in Japan: association with a nationwide palliative care education program. Cancer. 2018;124(3):626-35.

29. De Vleminck A, Houttekier D, Pardon $K$, et al. Barriers and facilitators for general practitioners to engage in advance care planning: a systematic review. Scand J Prim Health Care. 2013;31(4):215-26.

30. Fulmer T, Escobedo M, Berman A, Koren MJ, Hernández S, Hult A. Physicians' views on advance care planning and end-of-life care conversations. J Am Geriatr Soc. 2018;66(6):1201-5.

\section{Publisher's Note}

Springer Nature remains neutral with regard to jurisdictional claims in published maps and institutional affiliations.
Ready to submit your research? Choose BMC and benefit from:

- fast, convenient online submission

- thorough peer review by experienced researchers in your field

- rapid publication on acceptance

- support for research data, including large and complex data types

- gold Open Access which fosters wider collaboration and increased citations

- maximum visibility for your research: over $100 \mathrm{M}$ website views per year

At BMC, research is always in progress.

Learn more biomedcentral.com/submissions 